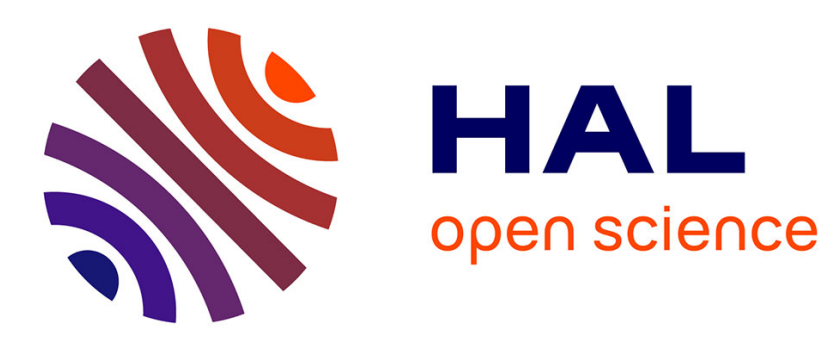

\title{
Rest shape computation for highly deformable model of brain.
}

Fanny Morin, Hadrien Courtecuisse, Matthieu Chabanas, Yohan Payan

\section{To cite this version:}

Fanny Morin, Hadrien Courtecuisse, Matthieu Chabanas, Yohan Payan. Rest shape computation for highly deformable model of brain.. Computer Methods in Biomechanics and Biomedical Engineering, 2015, 18 Suppl 1, pp.2006-2007. 10.1080/10255842.2015.1070591 . hal-01217673

\section{HAL Id: hal-01217673 \\ https://hal.science/hal-01217673}

Submitted on 5 Nov 2015

HAL is a multi-disciplinary open access archive for the deposit and dissemination of scientific research documents, whether they are published or not. The documents may come from teaching and research institutions in France or abroad, or from public or private research centers.
L'archive ouverte pluridisciplinaire HAL, est destinée au dépôt et à la diffusion de documents scientifiques de niveau recherche, publiés ou non, émanant des établissements d'enseignement et de recherche français ou étrangers, des laboratoires publics ou privés. 


\title{
Rest shape computation for highly deformable model of brain
}

\author{
F. Morin ${ }^{\mathrm{ab} *}$, H. Courtecuisse ${ }^{\mathrm{b}}$, M. Chabanas ${ }^{\mathrm{a}}$, and Y. Payan ${ }^{\mathrm{a}}$ \\ ${ }^{a}$ TIMC-IMAG, Univ. Grenoble Alpes, CNRS, F-38000 Grenoble, France \\ ${ }^{b}$ AVR-ICube, Univ. Strasbourg, CNRS, F-67000 Strasbourg, France
}

Keywords: biomechanical simulation; rest shape; collisions; brain model

\section{Introduction}

In medical simulations, finite element (FE) models are often extracted from medical images. This initial geometry usually corresponds to a state of the model at equilibrium between external and internal forces. Therefore, an important issue in biomechanics is to estimate the initial stress of FE model before running a simulation.

A typical example concerns gravity. Most biomechanical models ignore the gravity and directly run the simulation from the initial mesh with a null stress. However, this will obviously yield to different results, especially for living tissue that are usually soft and undergo large deformations. When the gravity force is applied to a model, it will shrink down unless other conditions are accounted for: boundary conditions, internal forces (e.g. muscle contraction) or other external forces or pressures. A rest shape must therefore be determined so that the model converges to the targeted equilibrium state when loads are applied.

Some methods propose to directly compute these loads whereas others prefer to estimate the rest shape of the model that will imply pre-stressed model at equilibrium. These inverse problems admit an exact formulation as a set of differential equations (Govindjee and Mihalic 1996) which has the benefit to find an exact and unique solution. However, the derivation of this formulation is cumbersome and depends on biomechanical laws of the model. On the other hand, Sellier (2011) proposed an iterative technic in order to compute rest position. That position may not be exact but its formulation doesn't rely on biomechanical laws.

In this paper, a method is presented in the context of a biomechanical model of the brain that will be used to model brain-shift during surgery. This phenomenon is mainly caused by a loss of cerebro-spinal fluid (CSF) surrounding brain. Our approach is based on Sellier (2011), with the addition of contact forces which enable the iterative method to converge towards an equilibrium state.

\section{Methods}

\subsection{Biomechanical model}

We used FE model with co-rotational approach (Müller et al. 2002) for the brain and collisions with skull are handled with Lagrangian Multipliers as explained in Courtecuisse et al. (2013).

Gravity and CSF pressure are applied on the model. CSF pressure is modeled as a uniform force field applied on the outer nodes of the mesh along their normal.

\subsection{Computation of the rest shape}

For this purpose, we extended the method proposed by Sellier (2011). With $X_{i}^{\text {ini }}$ the initial segmented position of node $i, X_{i}^{\text {tar }}$ the target position, we look for the rest position $X_{i}^{\text {rest }}$ so that the equilibrium state $X_{i}^{e q}$ is as close as possible to $X_{i}^{\text {tar }}$. Our algorithm is:

1) $\forall i, X_{i}^{\text {tar }}=X_{i}^{\text {rest }}=X_{i}^{\text {ini }}$

2) Simulate until equilibrium is reached, accounting for contacts

3) $\forall i, \Delta_{i}=X_{i}^{\text {tar }}-X_{i}^{e q}$

4) $\Delta=\frac{\sum\left\|\Delta_{i}\right\|}{\text { nb Nodes }}$

5) If $\Delta>\varepsilon, \forall i X_{i}^{\text {rest }}+\Delta_{i}$ and go to step (2) else accept $\left(X_{i}^{\text {rest }}\right)$ as the final rest positions.

In his method, Sellier (2011) proposed to compute $\Delta$ in step 4) as the maximum of the $\left\|\Delta_{i}\right\|$. But, this maximal value is often reached for few nodes set in highly constrained areas (such as contact regions or areas close to fixed points). Thus, computing the average value has the benefit to give a more global regularization to the model.

Moreover, as stated by Sellier (2011) in his proof of convergence, his method converges when deformations are small enough. In our case, due to the low stiffness of the brain, the biomechanical model undergoes large deformations when gravity and CSF pressure are applied. The model oscillates and finally diverges. Thus, no equilibrium state can be found.

Therefore, we propose in step 2) to account for collisions with the skull during this pre-stressing process. The normals of the skull contact surface are oriented toward the inside of the cavity, with collisions handled on direct orientation only. This means a node cannot move out of the skull boundaries but is allowed to cross it from the outside to the inside. This point is crucial since some of the rest positions computed with the above algorithm may be outside the skull.

*Corresponding author. Email: fanny.morin@imag.fr 
Handling such contacts limits the deformations of the biomechanical model within the skull boundaries, which finally enables the iterative algorithm to converge towards an equilibrium state.

\section{Results and discussion}

\subsection{Rest shape}

The brain model was segmented from MR images using ITK-SNAP (www.itksnap.org), including main anatomical parts such as tumor area, brainstem, cerebellum and skull. Then, the meshing step was completed with CGAL (www.cgal.org) which allows creating labelled tetrahedral FE meshes from segmentation images. Finally, the FE model was developed with the simulation framework Sofa (www.sofa-framework.org). This is illustrated in Figure 1.
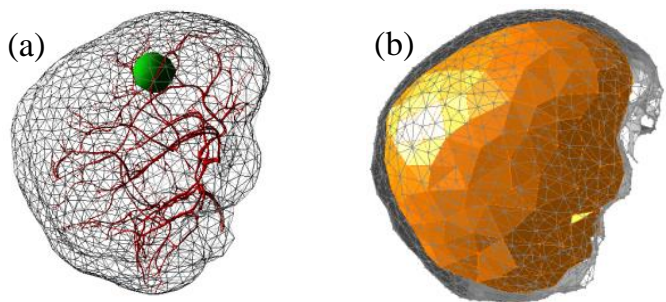

Figure 1: visual (a) and collision (b) models in Sofa

For the simulation, we used a FE mesh with 5266 elements and 1081 nodes. The Young modulus was fixed to $1.5 \mathrm{kPa}$ for the brain and $10 \mathrm{kPa}$ for the tumor area whereas Poisson ratio was uniformly set to 0.45 (Schiavone et al. 2009). Nodes of brainstem and cerebellum were fixed.

Rest shape (fig. 2(a)) was found in 6 iterations, with average error $\Delta$ equal to $0.746 \mathrm{~mm}$. Figure 2(b) shows that highest errors are located on the surface of the model, close to contact areas. Nevertheless, inside the volume, the errors are low and the shape is well interpolated.
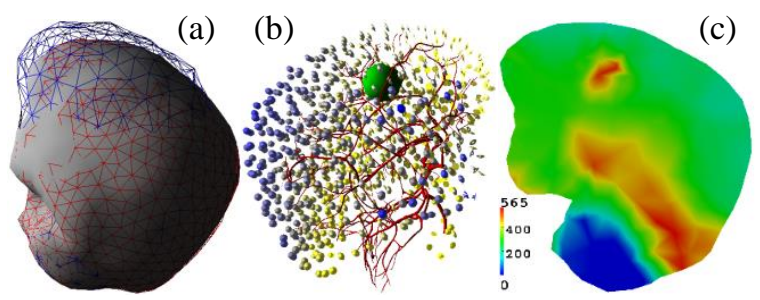

Figure 2: (a) simulated model (grey) with rest positions (blue) and target positions (red) (b) error repartition on FE mesh from yellow (close to $0 \mathrm{~mm}$ ) to dark blue $(2.69 \mathrm{~mm})(\mathrm{c})$ von Mises stress (in $\mathrm{Pa}$ ) on a slice of the mesh at equilibrium

Figure 2(c) shows von Mises stress repartition on a slice of the mesh at equilibrium. The blue area corresponds to the fixed cerebellum part: stress is quasi null here. Above this region, there is a red area with high stress due to the gravity acting on the mesh.
Finally, the heterogeneous region of the tumor also undergoes high stress because of its higher stiffness.

\subsection{Impact of the pre-stressing step}

In this section, we would like to show the importance of having pre-stressed models taking external forces into account.

On that purpose, we used two FE models. The first model was pre-stressed with our method, accounting for gravity and CSF pressure. Conversely, the second one was not pre-stressed, and thus, did not account for external forces. Then, we applied the same force field on each model.

Results show that both models deformed on the same direction but with different amplitude of displacement (Table 1): as expected, the pre-stressed model appears much stiffer than the other one.

\begin{tabular}{|l|c|c|}
\hline & Mean displ. & Max. displ. \\
\hline 1. Pre-stressed & $3.0347 \mathrm{~mm}$ & $6.9218 \mathrm{~mm}$ \\
\hline 2. Not pre-stressed & $4.7843 \mathrm{~mm}$ & $13.3968 \mathrm{~mm}$ \\
\hline Increase & $57.7 \%$ & $93.5 \%$ \\
\hline
\end{tabular}

Table 1: impact of an force field applied on prestressed (1) and not pre-stressed model (2)

\section{Conclusions}

This paper presents a method to compute rest shape for highly deformable models. This technique is based on Sellier (2011) algorithm, with the addition of collisions with a surface surrounding the simulated mesh. This was presented on a specific biomechanical model of the brain, nevertheless the method could be generalised to other contexts. Moreover, we also presented results showing the importance of taking external forces into account for medical biomechanical simulations.

\section{Acknowledgements}

The authors would like to thank Pr. O. Palombi from the department of Neurosurgery, Grenoble Hospital. This work was partly supported by the French ANR within the Investissements d'Avenir program (Labex CAMI) under reference ANR-11-LABX-0004.

\section{References}

Courtecuisse H, Allard J, Kerfriden P, Bordas SPA, Cotin S. 2013. Real-time simulation of contact and cutting of heterogeneous soft-tissues. Medical Image Analysis.

Govindjee S, Mihalic PA. 1996. Computational methods for inverse finite elastostatics. Computational Methods in Applied Mechanics and Engineering 136, 47-57.

Müller M, Dorsey J, McMillan L, Jagnow R, Cutler B. 2002. Stable real-time deformations. ACM SIGGRAPH/ Eurographics, 49-54.

Schiavone P, Chassat F, Boudou T, Promayon E, Valdivia F, Payan Y. 2009. In vivo measurement of human brain elasticity using a light aspiration device. Medical Image Analysis 13: 673-678.

Sellier M. 2011. An iterative method for the inverse elastostatic problem. Journal of Fluids and Structures 27:14611470. 